\title{
Comparison between using generalized differential quadrature method and analytical solution in analyzing vibration behavior of nonuniform nanobeam systems
}

\author{
Hessam Bakhshi Khaniki', Shahrokh Hosseini Hashemi ${ }^{2}$ Hossein Bakhshi Khaniki',* \\ ${ }^{1}$ Department of Industrial Engineering, Sharif University of Technology, Tehran, Iran \\ ${ }^{2}$ School of Mechanical Engineering, Iran University of Science and Technology, Tehran, Iran
}

\begin{abstract}
In this article, generalized differential quadrature method (GDQM) is used to study the free vibrational behavior of variable cross section nano beams. Eringen's nonlocal elastic theory is taken into account to model the small scale effects and nonuniformity is assumed by exponentially varying the width of nano beam. Governing equation of motion is solved using generalized differential quadrature method with different numbers of sampling points. Effects of increasing the sampling points in reaching more accurate results for first three frequency parameters are presented and it is shown that after a specific number of sampling points, results merge to a certain accurate number. It is concluded that generalized differential quadrature method is able to reach the correct answers comparing to analytical results. Moreover, due to the stiffness softening behavior of small-scale structures, necessity of using Eringen's nonlocal elastic theory to model the small scale effects due to the frequency variation is observed.
\end{abstract}

Keywords: Generalized differential quadrature method; GDQM; nanobeam; nonlocal beam; nonuniform beam; Variable cross section.

\section{Introduction}

Beams are one of the important structures used in most mechanical designs ever since. With the improvements done in new devices, scale of such structures have been reduced to micro/nano in order to reach a smaller and lighter machines. In this study, generalized differential quadrature method is used to solve the governing equations of nonuniformnanobeams. Scale effect is defined using Eringen's nonlocal elastic theory and beam is modeled using Euler-Bernoulli beam theory. Nonuniformity is proposed using exponential width variation while thickness remains constant through the beam in which a schematic model is presented in Fig. 1 and used is previous studies (Ece et al. 2007, Khaniki and Hashemi 2016, Hashemi and Khaniki 2017).

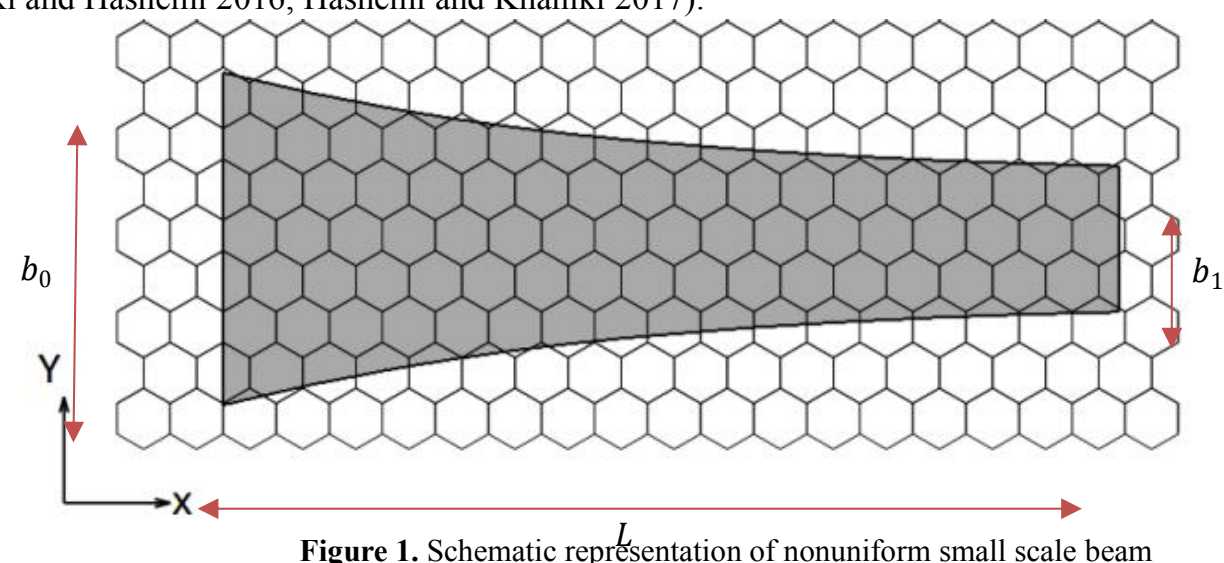

\section{Problem formulation}

General equation of motion for nonlocal Euler-Bernoulli nonuniformnanobeam is achieved as:

Copyright (C) 2018 Hessam Bakhshi Khaniki et al.

doi: 10.24294/can.v1i2.202

EnPress Publisher LLC.This work is licensed under the Creative Commons Attribution-NonCommercial 4.0 International License (CC BY-NC 4.0).

http://creativecommons.org/licenses/ by/4.0/ 


$$
E I \frac{d^{4} w}{d x^{4}}+2 E \frac{d I}{d x} \frac{d^{3} w}{d x^{3}}+E \frac{d^{2} I}{d x^{2}} \frac{d^{2} w}{d x^{2}}+\left(e_{0} a\right)^{2} \rho A \omega^{2} \frac{d^{2} w}{d x^{2}}+2\left(e_{0} a\right)^{2} \rho \frac{d A}{d x} \omega^{2} \frac{d w}{d x}+\left(e_{0} a\right)^{2} \rho \frac{d^{2} A}{d x^{2}} \omega^{2} w-\rho A \omega^{2} w=0
$$

where $\mathrm{E}$ is the Young's modulus term, I and A are the second moment inertia and cross section terms, $\mathrm{e}_{0} \mathrm{a}$ are the small scale parameters, $\omega$ is the frequency term and $\rho$ is the mass density. In this study as mentioned before, exponential variation is presented for width of nonuniformnanobeam with constant thickness. Therefore nonuniformity terms are presented as:

$$
A(x)=b_{0} h e^{N x}, I(x)=\frac{1}{12} b_{0} h^{3} e^{N x}
$$

where $b_{0}$ is the width of the beam at $\mathrm{x}=0, \mathrm{~h}$ is the thickness of the beam and $\mathrm{N}$ is the exponential nonuniformity term and by defining new parameters to have same range of scales between terms as:

$$
X=x / L, \quad Y=y / \mathrm{L}, \quad \eta=N L, \quad \alpha=e_{0} a / L, \quad \lambda^{2}=\frac{\rho A L^{4} \omega^{2}}{E I}
$$

in which by using these new parameters and nonuniformity terms, equation of motion of nonuniformnanobeam could be rewritten in nondimensional form as:

$$
\frac{d^{4} W}{d X^{4}}+2 \eta \frac{d^{3} W}{d X^{3}}+\left(\eta^{2}-\alpha^{2} \lambda^{2}\right) \frac{d^{2} W}{d X^{2}}-\alpha^{2} \eta \lambda^{2} \frac{d W}{d X}+\lambda^{2}\left(1-\alpha^{2} \eta^{2}\right) W=0
$$

where $\lambda$ denotes the nondimensional frequency term.

\section{Solution Procedure}

As mentioned before, in order to solve the nondimensional equation of motion of nonuniformnanobeam presented in Eq.(5), generalized differential quadrature method (GDQM) is employed. This theory uses monomials coefficients and sampling points to introduce the deflection variable as:

$$
W^{(r)}\left(X_{i}\right)=\sum_{k=0}^{1} h_{1 k}^{(r)}\left(X_{i}\right) W_{1}^{(\mathrm{k})}+\sum_{j=2}^{N-1} h_{j 0}^{(r)}\left(X_{i}\right) W_{j}+\sum_{k=0}^{1} h_{N k}^{(r)}\left(X_{i}\right) W_{N}^{(\mathrm{k})}=\sum_{k=1}^{N+2} E_{i k}^{(r)} U_{k}
$$

where $E_{i k}$ represent the monomials coefficients and $N$ is the number of sampling points. $h_{i k}^{(r)}$ are the Hermite interpolation shape functions as:

$$
\left\{\begin{array}{l}
h_{j l}^{(r)}\left(X_{i}\right)=\delta_{i j} \delta_{l r} \\
h_{p i}=(X-1)^{2}\left(a_{p i} X^{2}+b_{p i} X+C_{p i}\right) l_{p}(X), \quad p=1, N ; i=0,1,2 \\
h_{j 0}=\left[X^{2}(X-1)^{2} / X_{j}{ }^{2}\left(X_{j}-1\right)^{2}\right] l_{j}(X) \quad j=2,3, \ldots, N-1
\end{array}\right.
$$

and $l_{i}$ are the off diagonal terms defined as:

$$
\begin{gathered}
l_{i k}^{(1)}=\frac{\prod_{v=1, v \neq i}^{N}\left(X_{i}-X_{v}\right)}{\left(X_{i}-X_{k}\right) \prod_{v=1, v \neq k}^{N}\left(X_{k}-X_{v}\right)} \quad i, k=12,3, \ldots, N \text { and } k \neq i \\
l_{i k}^{(\mathrm{r})}=r\left[A_{i i}^{(\mathrm{r}-1)} A_{i k}^{(1)}-\frac{A_{i k}^{(\mathrm{r}-1)}}{x_{i}-x_{k}}\right] \quad i, k=12,3, \ldots, N \text { and } k \neq i, r>1
\end{gathered}
$$

and $\mathrm{a}_{\mathrm{pi}}, \mathrm{b}_{\mathrm{pi}}$ andc $\mathrm{p}_{\mathrm{pi}}$ are the coefficients defined as

$$
\begin{aligned}
& \left\{\begin{array}{l}
a_{10}=\left(l_{1}^{(1)}\left(X_{1}\right)+2\right)^{2}-\frac{l_{1}^{(2)}\left(X_{1}\right)}{2}-1+2 l_{1}^{(1)}\left(X_{1}\right) \\
a_{11}=-\left(l_{1}^{(1)}\left(X_{1}\right)+2\right)^{2} \\
a_{12}=\frac{1}{2}
\end{array}\right. \\
& \left\{\begin{array}{l}
b_{10}=-\left(l_{1}^{(1)}\left(X_{1}\right)+2\right)-2 a_{10} X_{1} \\
b_{11}=1-2 a_{11} X_{1} \\
b_{12}=-X_{1}
\end{array}\right.
\end{aligned}
$$




$$
\begin{aligned}
& \left\{\begin{array}{l}
c_{10}=1+a_{10} X_{1}^{2}+X_{1}\left(l_{1}^{(1)}\left(X_{1}\right)+2\right) \\
c_{11}=X_{1}\left(a_{11} X_{1}-1\right) \\
c_{12}=\frac{X_{1}^{2}}{2}
\end{array}\right. \\
& \left\{\begin{array}{l}
a_{N 0}=\left(l_{N}^{(1)}\left(X_{N}\right)-2\right)^{2}-\frac{l_{N}^{(2)}\left(X_{N}\right)}{2}-1+2 l_{N}^{(1)}\left(X_{N}\right) \\
a_{N 1}=-\left(l_{N}^{(1)}\left(X_{N}\right)+2\right)^{2} \\
a_{N 2}=\frac{1}{2}
\end{array}\right. \\
& \left\{\begin{array}{l}
b_{N 0}=-\left(l_{N}^{(1)}\left(X_{N}\right)+2\right)-2 a_{N 0} X_{N} \\
b_{N 1}=1-2 a_{N 1} X_{N} \\
b_{N 2}=-X_{N} \\
c_{N 0}=1+a_{N 0} X_{N}^{2}+X_{N}\left(l_{N}^{(1)}\left(X_{N}\right)+2\right) \\
c_{N 1}=X_{N}\left(a_{N 1} X_{N}-1\right) \\
c_{N 2}=\frac{X_{N}^{2}}{2}
\end{array}\right.
\end{aligned}
$$

In this study, boundary conditions are assumed to be simply-supported therefore the boundary equations are achieved as:

$$
\text { Simply-Supported : } W\left(X_{i}\right)=0, \frac{d^{2} W\left(X_{i}\right)}{d X^{2}}=0 \quad i=1 \& N
$$

It should be mentioned that although this solution is done for simply-supported nonuniformnanobeams but it could be also assigned to other kinds of boundaries. Using these equations and rewriting Eq. (4) leads to

$$
\sum_{k=1}^{N+2}\left(E_{i k}^{(4)}+2 \eta E_{i k}^{(3)}+\eta^{2} E_{i k}^{(2)}\right) U_{k}-\lambda^{2} \sum_{k=1}^{N+2}\left(\alpha^{2} E_{i k}^{(2)}+\alpha^{2} \eta E_{i k}^{(1)}-\left(1-\alpha^{2} \eta^{2}\right) E_{i k}\right) U_{k}=0
$$

where $E_{i k}$ could be defined using previous literatures (Wu and Liu 1999 and 2000). Equations achieved for each sampling point in Eq. (16) could be written in a matrix form as:

$$
\left[\begin{array}{cc}
{\left[S_{b b}\right]_{4 \times 4}} & {\left[S_{b d}\right]_{4 \times 4}} \\
{\left[S_{d b}\right]_{(n-1) \times(n-1)}} & {\left[S_{d d}\right]_{(n-1) \times(n-1)}}
\end{array}\right]\left\{\begin{array}{l}
\left\{U_{b}\right\} \\
\left\{U_{d}\right\}
\end{array}\right\}-\lambda^{2}\left[\begin{array}{cc}
{[0]_{4 \times 4}} & {[0]_{4 \times 4}} \\
{\left[Q_{d b}\right]_{(n-1) \times(n-1)}} & {\left[Q_{d d}\right]_{(n-1) \times(n-1)}}
\end{array}\right]\left\{\begin{array}{l}
\left\{U_{b}\right\} \\
\left\{U_{d}\right\}
\end{array}\right\}=0
$$

and accordingly, generalized eigenvalue equation could be reached as:

$$
\left[S_{d d}\right]-\left[S_{d b}\right]\left[S_{b b}\right]^{-1}\left[S_{b d}\right]-\lambda^{2}\left[Q_{d d}\right]-\left[Q_{d b}\right]\left[S_{b b}\right]^{-1}\left[S_{b d}\right]=0
$$

By calculating the results for Eq. (18), frequency mode numbers are achieved.

\section{Results and Discussion}

As shown in previous sections, free vibration equation of motion of nonuniform nonlocal beam is presented and solution process using GDQ method is obtained step by step. In order to study the accuracy of current solution process and methodology, frequency terms are obtained for different numbers of sampling points and compared to those calculated using analytical solution. For this reason, first three nondimensional frequency terms are calculated and presented in Table 1. Different number of sampling points is chosen which is varied from 5 to 8 sample points. Moreover, average error with respect to analytical results obtained by Hashemi and Khaniki (2017) is calculated and presented. It can be seen that increasing the number of sampling points reduces the average related errors. After a number of sampling points, results merge to specific numbers which compared to analytical ones, are in a great agreement. From the results once again it is seen that increasing the nonlocal term reduces the frequency term in all first three modes of vibration.

\begin{tabular}{|l|c|c|c|c|c|l|}
\hline Solution Method & $\alpha=\mathbf{0 . 4}$ & $\alpha=\mathbf{0 . 3}$ & $\alpha=\mathbf{0 . 2}$ & $\alpha=\mathbf{0 . 1}$ & $\alpha=\mathbf{0 . 0}$ & error(\%) \\
\hline Analytical & 2.624137 & 2.787796 & 2.948800 & 3.076059 & 3.126165 & - \\
\hline
\end{tabular}




\begin{tabular}{|l|l|l|l|l|l|l|}
\hline $\begin{array}{l}\text { (Hashemi and Khaniki } \\
\text { 2017) }\end{array}$ & & & & & & \\
\hline GDQM 5 Nodes & 2.648128 & 2.790631 & 2.975591 & 3.103779 & 3.141507 & $6.63278 \mathrm{e}-3$ \\
\hline GDQM 6 Nodes & 2.623994 & 2.787297 & 2.948568 & 3.077145 & 3.127054 & $1.89918 \mathrm{e}-4$ \\
\hline GDQM 7 Nodes & 2.624128 & 2.787754 & 2.948798 & 3.076066 & 3.126171 & $4.67371 \mathrm{e}-6$ \\
\hline GDQM 8 Nodes & 2.624128 & 2.787754 & 2.948798 & 3.076066 & 3.126171 & $4.67371 \mathrm{e}-6$ \\
\hline
\end{tabular}

Table 1. First mode of vibration frequency term in nonuniform nanobeam with $\eta=1$

\begin{tabular}{|l|l|l|l|l|l|l|}
\hline Solution Method & $\alpha=0.4$ & $\alpha=0.3$ & $\alpha=0.2$ & $\alpha=0.1$ & $\alpha=0.0$ & error(\%) \\
\hline $\begin{array}{l}\text { Analytical } \\
\text { (Hashemi and Khaniki } \\
\text { 2017) }\end{array}$ & 3.795550 & 4.315183 & 5.004425 & 5.820408 & 6.290498 & - \\
\hline GDQM 5 Nodes & 3.810800 & 4.321869 & 5.005180 & 5.840437 & 6.302245 & 2.20534 e-3 \\
\hline GDQM 6 Nodes & 3.796457 & 4.3160276 & 5.005812 & 5.821824 & 6.292014 & $2.39225 \mathrm{e}-4$ \\
\hline GDQM 7 Nodes & 3.795564 & 4.315108 & 5.004471 & 5.820398 & 6.290454 & $7.79473 \mathrm{e}-6$ \\
\hline GDQM 8 Nodes & 3.795564 & 4.315108 & 5.004471 & 5.820398 & 6.290454 & $7.79473 \mathrm{e}-6$ \\
\hline
\end{tabular}

Table 2. Second mode of vibration frequency term in nonuniform nanobeam with $\eta=1$

\begin{tabular}{|l|l|l|l|l|l|l|}
\hline Solution Method & $\alpha=0.4$ & $\alpha=0.3$ & $\alpha=0.2$ & $\alpha=0.1$ & $\alpha=0.0$ & error(\%) \\
\hline $\begin{array}{l}\text { Analytical } \\
\text { Hashemi and Khaniki } \\
\text { 2017) }\end{array}$ & 4.768500 & 5.442014 & 6.467170 & 8.073308 & 9.432419 & - \\
\hline GDQM 5 Nodes & 4.767854 & 5.441456 & 6.466045 & 8.072585 & 9.430874 & $1.33063 \mathrm{e}-4$ \\
\hline GDQM 6 Nodes & 4.768421 & 5.442874 & 6.466924 & 8.072987 & 9.432010 & $5.91514 \mathrm{e}-5$ \\
\hline GDQM 7 Nodes & 4.768472 & 5.441987 & 6.467122 & 8.073358 & 9.432371 & $5.90749 \mathrm{e}-6$ \\
\hline GDQM 8 Nodes & 4.768472 & 5.441987 & 6.467122 & 8.073358 & 9.432371 & $5.90749 \mathrm{e}-6$ \\
\hline
\end{tabular}

Table 3. Third mode of vibration frequency term in nonuniform nanobeam with $\eta=$

\section{Conclusion}

Free vibration analysis of variable cross section nonlocal small scale beam is done using GDQ method. Nanobeam is modeled using Euler-Bernoulli beam and Eringen's nonlocal elastic theory. Generalized differential quadrature method is presented step by step for the current problem. Calculation is done for different number of sampling points and results for first three frequency parameters are presented and compared to those achieved by analytically solving the problem. Average related error is presented for different number of sampling point and accuracy of current method is discussed. It is shown that GDQM method has a great ability and accuracy in solving this problem formulation and frequency term is achieved with high accuracy. It is mentioned that increasing the nonlocal parameter in exponential cross section variable nonuniformnanobeam leads to smaller frequency parameters in all the first three mode shapes using simply support as boundary conditions and it is necessary to add nonlocal effects in analyzing nonuniformnanobeams.

\section{References}

1. Ece MC, Aydogdu M, Taskin V. Vibration of a variable cross-section beam. Mechanics Research Communications 2007; 34(1): 78-84.

2. Khaniki HB, Hashemi SH. Free Vibration Analysis of Nonuniform Microbeams Based on Modified Couple Stress Theory: An Analytical Solution. International Journal of Engineering-Transactions B: Applications 2017; 30(2): 311-320.

3. Hashemi SH, Khaniki HB. Analytical solution for free vibration of a variable cross-section nonlocal nanobeam. International Journal of Engineering-Transactions B: Applications 2016; 29(5): 688-696.

4. Hashemi SH, Khaniki HB. Vibration analysis of a timoshenko non-uniform nanobeam based on nonlocal theory: An analytical solution. International Journal of Nano Dimension. 2017; 8(1): 70-81. 
5. Hashemi SH, Khaniki HB. Free Vibration Analysis of Functionally Graded Materials Non-uniform Beams. International Journal of Engineering-Transactions C: Aspects 2016;29(12): 1734-1740.

6. Wu TY, Liu GR. A differential quadrature as a numerical method to solve differential equations. Computational Mechanics 1999; 24(3): 197-205.

7. Wu TY, Liu GR. Application of generalized differential quadrature rule to sixth - order differential equations. International Journal for Numerical Methods in Biomedical Engineering 2000; 16(11): 777-784. 